\title{
Prosthetic Aortic Valve Endocarditis in a Patient With Birt- Hogg-Dube Syndrome due to Lactobacillus paracasei
}

\author{
Mustafa Ajam ${ }^{\mathrm{a}, \mathrm{c}}$, Omeralfaroug Adam ${ }^{\mathrm{a}}$, Ahmed Yeddi ${ }^{\mathrm{a}}$, Mowyad Kahlid ${ }^{\mathrm{a}}$, \\ Mohamed Shokr ${ }^{b}$, Luis Afonso ${ }^{b}$
}

\begin{abstract}
Various causative organisms and predisposing factors were implicated in the pathogenic process behind prosthetic valve endocarditis. Lactobacillus paracasei, a classic constituent of probiotics, rarely causes infections in humans and was reported only in few case reports. The use of probiotics was hypothesized to be a risk factor for these infections; however, no causative relationship could be drawn. We describe a 75-year-old woman with history of Birt-Hogg-Dube syndrome and bioprosthetic aortic valve replacement who presented with worsening dyspnea was found to have Lactobacillus paracasei bacteremia and evidence of bacterial vegetations noted on transesophageal echocardiography (TEE) along with evidence of severe aortic insufficiency. Based on antibiotics sensitivity profile, she was treated with penicillin and gentamycin, which resulted in bacterial clearance on repeat blood cultures, and the patient was transferred to a different facility to undergo replacement of the prosthetic aortic valve. Although Lactobacillus paracasei is rarely involved in human infections, it should be suspected in patients with underlying structural heart/valvular disease, recent antibiotic exposure or recent probiotic use who are presenting with evidence of infective endocarditis. Combination antibiotics including beta lactams, aminoglycosides and clindamycin are suggested treatment of choice for this organism.
\end{abstract}

Keywords: Lactobacillus paracasei; Infective endocarditis; Prosthetic valve endocarditis; Probiotics

\section{Introduction}

In spite of the recent advances in the diagnostic and therapeutic approaches to infective endocarditis, it remains a major complication of prosthetic valve replacement, imposing sig-

Manuscript submitted June 13, 2019, accepted June 24, 2019

\footnotetext{
aDepartment of Internal Medicine, Detroit Medical Center/Wayne State University, Detroit, MI, USA

bDivision of Cardiology, Department of Internal Medicine, Detroit Medical Center/Wayne State University, Detroit, MI, USA

${ }^{\mathrm{c} C}$ Corresponding Author: Mustafa Ajam, Department of Internal Medicine, Detroit Medical Center/Wayne State University, 4201 St. Antoine, Detroit, MI, USA.Email: m_ijam@yahoo.com
}

doi: https://doi.org/10.14740/cr901 nificant high rates of morbidity and mortality. Prosthetic valve endocarditis can be classified into early onset, developing within the first 60 days following the procedure, intermediate onset if infection occurs within 60 - 365 days and late onset when it develops after the first year. Staphylococcus aureus and coagulase-negative Staphylococci are the most commonly reported organisms, particularly within the first year after the valve replacement, while infection rates due to Enterococci and viridans Streptococci rise afterwards [1].

Lactobacillus, a normal constituent of the gastrointestinal (GI) and genitourinary tracts bacterial flora, is an anaerobic or facultatively anaerobic gram-positive rod-shaped bacteria. Although it rarely causes infections in humans, Lactobacillus bacteremia has been reported in patients with underlying medical conditions including cancer, diabetes mellitus, recent surgery or those with recent exposure to antibiotics. A wide range of infections have been described, including infective endocarditis, meningitis and intra-abdominal abscesses [2].

Lactobacillus rhamonsus and Lactobacillus casei are the most commonly reported species that are involved in human infections. Lactobacillus paracasei, another member of this family, is rarely encountered and is most commonly used in probiotics [3].

To the best of our knowledge, there are four previously reported cases of infective endocarditis due to Lactobacillus paracase $i$ in the literature [3-6]. In this report, we highlight a rare presentation of this rare organism. We believe that it will add to the level of awareness regarding Lactobacillus paracasei infective endocarditis, possible sources of infection and the selection of appropriate antibiotics.

\section{Case Report}

A 75-year-old woman with a history of Birt-Hogg-Dube syndrome (a rare autosomal dominant syndrome characterized by various skin manifestations, cystic lung disease and predisposition to renal cell carcinoma [7]), severe aortic stenosis requiring valve replacement with bioprosthetic valve 9 years prior to presentation and history of hypothyroidism, presented with shortness of breath for 2 days. Her vital signs on admission were showing a heart rate of 114 beats per minute (BPM), blood pressure of $167 / 99 \mathrm{~mm} \mathrm{Hg}$, respiratory rate of 28 breaths per min, oxygen saturation of $89 \%$ on pulse oximetry and temperature of $36.6^{\circ} \mathrm{C}$. Physical examination revealed 


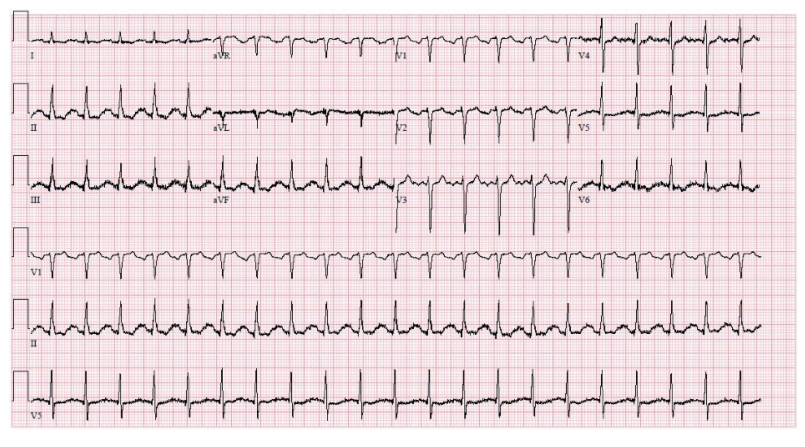

Figure 1. EKG on admission showing sinus tachycardia at a rate of $127 \mathrm{BPM}$.

poor bilateral lung air entry and a systolic murmur loudest at the left sternal border. Blood work revealed acute hypoxic hypercapnic respiratory failure on blood gas analysis, elevated brain natriuretic peptide (BNP) levels at $288 \mathrm{pg} / \mathrm{mL}$ (normal $<101 \mathrm{pg} / \mathrm{mL}$ ), elevated lactic acid levels along with elevated white cell count (WBC) of 22,600 cells $/ \mu \mathrm{L}$ (normal 3,500 10,500 cells $/ \mu \mathrm{L}$ ) and neutrophils count of 18,000 cells $/ \mu \mathrm{L}$ (normal 1,580 - 7,130 cells/ $\mu \mathrm{L}$ ). Initial troponin levels were negative $(<0.04 \mathrm{ng} / \mathrm{mL})$; however, it increased to $0.25 \mathrm{ng} / \mathrm{mL}$ after $24 \mathrm{~h}$ then trended down to $0.19 \mathrm{ng} / \mathrm{mL}$. Soon after that, the patient was intubated in light of her acute respiratory failure and altered mental status. Workup for infectious etiology was initiated and two sets of blood cultures were obtained for organism identification. Her chest X-ray did not show cardiomegaly; however, it showed chronic bullous emphysematous changes and hyperinflated lungs (likely related to Birt-HoggDube syndrome) and possible superimposed edema. Her electrocardiogram $(\mathrm{EKG})$ revealed sinus tachycardia at a rate of 127 BPM without evidence of acute coronary syndrome (Fig. 1).

Broad spectrum intravenous (IV) antibiotics including vancomycin, cefepime, azithromycin, and metronidazole were initiated in the beginning and were subsequently changed to penicillin and gentamycin after obtaining the results of blood cultures which grew Lactobacillus paracasei in four sets. This regimen was guided by the antibiotic sensitivity profile that revealed sensitivity to penicillin, clindamycin, and gentamycin. Repeat blood cultures confirmed clearance of the bacteremia on this antibiotic regimen.

Transthoracic echocardiography (TTE) revealed normal left ventricular ejection fraction of $55 \%-60 \%$, bioprosthetic aortic valve and aortic regurgitant jet pressure halftime of 177 $\mathrm{ms}$, indicative of severe aortic regurgitation (severe aortic regurgitation $<250 \mathrm{~ms}$ ). However, no definitive comment could be made on the presence or absence of valvular vegetations.

TEE was done the following day and it showed a small filamentous mobile echogenicity measuring $0.9 \times 0.3 \mathrm{~cm}$ attached to the left coronary cusp of the aortic valve suggestive of a vegetation, in addition a small area of echo-lucency noted within the body of non-coronary cusp suggestive of perforation (Fig. 2; Supplementary Video 1).

In the meantime, workup for the source of bacteremia included an abdominal ultrasound that was concerning for acute cholecystitis with apparent multiple gallstones, mildly thick-

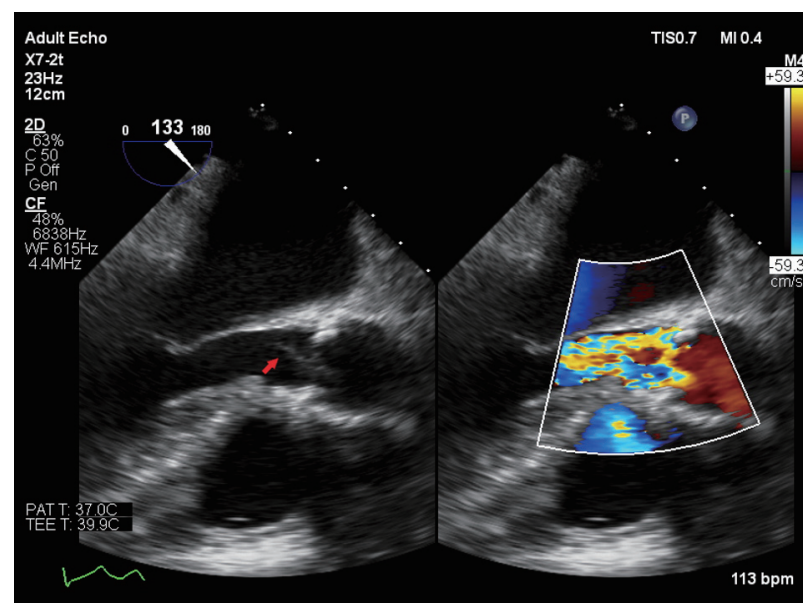

Figure 2. TEE mid-esophageal long axis view showing aortic valve vegetation (arrow) and aortic valve regurgitation on color flow Doppler.

ened gallbladder wall and a small amount of pericholecystic fluid. The surgical team decided to delay cholecystectomy until after surgical correction of her severe aortic regurgitation that was recommended by the cardiology and cardiothoracic teams. The patient was transferred to another facility for further evaluation of surgical correction/replacement of the aortic valve. After arrival at that facility, the cardiothoracic team recommended to continue medical management until further stabilization of her ongoing sepsis prior to any surgical intervention. Unfortunately, the patient sustained an accidental fall that resulted in head trauma and intra-cranial hemorrhage and death subsequently.

\section{Discussion}

Bioprosthetic valves carry a higher risk of bacterial endocarditis as compared to other types of mechanical valves, likely related to higher rates of tissue degeneration. Various other key factors play a major role in endocarditis development, particularly, the ability of the causative organism to adhere and grow on the valve cusps mediated by different molecular interactions between bacterial and valvular surfaces that can establish the hosting media for bacterial colonization. Staphylococcus aureus, coagulase-negative Staphylococci and certain types of Streptococci exert a unique ability to establish these steps [1].

On the other hand, Lactobacilli have a lesser ability to produce infective endocarditis. In fact, animal studies revealed that Lactobacilli infectivity is 10 to 100 times less than that of Staphylococcus aureus and Streptococci [8], which in part, can explain its predominance among patients with severe underlying medical conditions or those with underlying structural heart disease, which may facilitate colonization of low virulence bacteria. Lactobacillus casei is the most commonly reported species of this family to cause infective endocarditis. Lactobacillus rhamnosus is another reported species that is implicated in human infections. However, Lactobacillus paracasei is not commonly encountered [9]. Dellaglio et al [8] 
suggested that Lactobacillus paracasei and casei are members of the same taxon and can be both viewed as the same species, which can shed the light on possible hidden cases of Lactobacillus paracasei infective endocarditis that has been reported as Lactobacillus casei.

There are four previous cases of Lactobacillus paracasei infective endocarditis reported in the literature [3-6]. In all four cases, there was an underlying structural heart disease including severe aortic and mitral stenosis, mitral and tricuspid insufficiency and even complicated ventricular septal defects (VSDs) and myocardial fibrosis. Some of the proposed risk factors included the use of probiotics and GI tract manipulation $[3,4]$. Treatment with IV $\beta$-lactams antibiotics, specifically penicillin and amoxicillin, and aminoglycosides or clindamycin was the treatment of choice in all four cases [3-6], and the use of chloramphenicol has been suggested in cases of resistance. Given the rarity of this condition, there are no Infectious Disease Society of America (IDSA) guidelines for the management of Lactobacillus endocarditis [10].

When reviewing 129 cases of Lactobacillus bacteremia, Canon et al [11] reported malignancy as the most common underlying condition, followed by diabetes mellitus and previous antibiotic exposure. In the same study, 73 cases of Lactobacillus endocarditis were identified, most commonly attributed to casei species, followed by rhamnosus and plantrum species. Significant risk factors noted in this subgroup included the presence of underlying structural heart disease, previous episodes of infective endocarditis, exposure to dental procedures and heavy dairy consumption.

Sources of entry of Lactobacilli are likely originating from the GI and genitourinary tracts. In a review of 45 cases of Lactobacillus bacteremia, Husni et al [2] noticed that $38 \%$ of those patients underwent abdominal or endoscopic procedures prior to the bacteremia onset. Furthermore, prior antibiotic exposure is another risk factor, resulting in the selective growth of Lactobacilli in the GI tract. Franko et al [3] also reported Lactobacillus paracasei infective endocarditis following colonoscopy in a consumer of probiotics, casting doubt on the safety of using probiotics and suggesting discontinuation of probiotics before any GI surgery or colonoscopy procedures. Although our patient did report using herbal supplements and eastern medicine, the exact name/brand and dose could not be identified, and no causal relationship between probiotics use and Lactobacillus infections could be drawn from our case or from reported cases in literature. Further research is needed for more understanding of the safety profile of probiotics and their implications in such infections.

\section{Conclusions}

Lactobacillus species can be an exceptionally rare cause of endocarditis, which is most commonly found in patients with severe underlying clinical conditions such as malignancy combined with underlying structural heart disease that is most consistent of severe valvular lesions. Even though it is exceptionally rare, we believe that it should be considered in any patient with endocarditis on top of structural heart abnormalities and especially in patients with prosthetic valves.

\section{Acknowledgments}

None.

\section{Financial Disclosure}

No extramural funding was used to support this work.

\section{Conflict of Interest}

None.

\section{Informed Consent}

Informed consent was obtained from the patient's family.

\section{Author Contributions}

MA, OA, AY, MK and MS contributed to drafting the manuscript; LA contributed to revision and final approval.

\section{References}

1. Avish Nagpal MRSJMS. Prosthetic valve endocarditis: state of the heart. Clin Invest. 2012;2(8):803-817.

2. Husni RN, Gordon SM, Washington JA, Longworth DL. Lactobacillus bacteremia and endocarditis: review of 45 cases. Clin Infect Dis. 1997;25(5):1048-1055.

3. Franko B, Vaillant M, Recule C, Vautrin E, Brion JP, Pavese P. Lactobacillus paracasei endocarditis in a consumer of probiotics. Med Mal Infect. 2013;43(4):171173.

4. Kato K, Funabashi N, Takaoka H, Kohno H, Kishimoto T, Nakatani Y, Matsumiya G, et al. Lactobacillus paracasei endocarditis in a consumer of probiotics with advanced and severe bicuspid aortic valve stenosis complicated with diffuse left ventricular mid-layer fibrosis. Int J Cardiol. 2016;224:157-161.

5. Schmidt V, Wolter M, Lenschow U, Kienast W. [Lactobacillus paracasei endocarditis in an 18-yeard-old patient with trisomy 21, atrioventricular septal defect and Eisenmenger complex: therapeutic problems]. Klin Padiatr. 2001;213(1):35-38.

6. Soleman N, Laferl H, Kneifel W, Tucek G, Budschedl E, Weber H, Pichler H, et al. How safe is safe?-a case of Lactobacillus paracasei ssp. paracasei endocarditis and discussion of the safety of lactic acid bacteria. Scand J Infect Dis. 2003;35(10):759-762.

7. Jensen DK, Villumsen A, Skytte AB, Madsen MG, Sommerlund M, Bendstrup E. Birt-Hogg-Dube syndrome: 
a case report and a review of the literature. Eur Clin Respir J. 2017;4(1):1292378.

8. Dellaglio F, Felis GE, Torriani S. The status of the species Lactobacillus casei (Orla-Jensen 1916) Hansen and Lessel 1971 and Lactobacillus paracasei Collins et al. 1989. Request for an opinion. Int J Syst Evol Microbiol. 2002;52(Pt 1):285-287.

9. Vankerckhoven V, Moreillon P, Piu S, Giddey M, Huys G, Vancanneyt M, Goossens H, et al. Infectivity of Lactobacillus rhamnosus and Lactobacillus paracasei isolates in a rat model of experimental endocarditis. J Med Microbiol.
2007;56(Pt 8):1017-1024.

10. Baddour LM, Wilson WR, Bayer AS, Fowler VG, Jr., Tleyjeh IM, Rybak MJ, Barsic B, et al. Infective endocarditis in adults: diagnosis, antimicrobial therapy, and management of complications: a scientific statement for healthcare professionals from the American Heart Association. Circulation. 2015;132(15):1435-1486.

11. Cannon JP, Lee TA, Bolanos JT, Danziger LH. Pathogenic relevance of Lactobacillus: a retrospective review of over 200 cases. Eur J Clin Microbiol Infect Dis. 2005;24(1):31-40. 